Intern. J. Fuzzy Mathematical Archive

Vol. 11, No.2, 2016, 53-62

ISSN: 2320-3242 (P), 2320-3250 (online)

Published on 30 November 2016

www.researchmathsci.org

DOI: http://dx.doi.org/10.22457/ijfma.v11n2a1

\title{
Common Fixed Point Theorems in Fuzzy 2 and Fuzzy 3-Metric Spaces
}

\author{
Preeti Sengar ${ }^{1}$, Suman Jain ${ }^{2}$, Aklesh Paria ${ }^{3}$ and V.H.Badshah ${ }^{4}$ \\ ${ }^{1,4}$ School of Studies in Mathematics, Vikram University, Ujjain (M.P.), India \\ ${ }^{2}$ Department of Mathematics, Govt. College, Kalapipal (M.P.), India \\ ${ }^{3}$ Department of Applied Mathematics, Medi-caps University Indore (M.P.), India \\ Email: preeti.sengar89@gmail.com
}

Received 21 September 2016; accepted 10 October 2016

\begin{abstract}
The purpose of this paper is to prove some common fixed point theorems in fuzzy-2, fuzzy-3 metric spaces by employing the notion of semi-compatible and reciprocal continuity. We extend and generalize the result of Chauhan et al. [1] and Som [19] from fuzzy metric spaces to fuzzy-2 metric and fuzzy-3 metric Spaces.
\end{abstract}

Keywords: Common fixed Point, Fuzzy metric space, fuzzy -2 metric spaces, fuzzy-3 metric spaces, semi-compatible, reciprocal continuity.

AMS Mathematics Subject Classification (2010): 47H10, 54H25

\section{Introduction}

The concept of fuzzy sets was introduced by Zadeh [20]. Kramosil and Michalek [10] introduced the concept of fuzzy metric space. George and Veeramani [7] modified this concept of fuzzy metric space and defined Hausdroff topology on fuzzy metric space. Many authors have studied common fixed point theorems in fuzzy metric spaces. The most interesting results in this direction are due to Cho [2], George and Veeramani [7], Grabiec [8], Kaleva [9], Kramosil and Michalek [10], Mishra et al. [11] etc., Singh and Chauhan [15] introduced the concept of compatibility in fuzzy metric space and proved some common fixed point theorems in fuzzy metric space.

Gahler [5-6] investigated 2-metric spaces in a series of his papers. It is to be remarked that Sharma, Sharma and Iseki [14] studied for the first time contraction type mappings in 2-metric spaces. In 2002, Sharma [13] introduced fuzzy 2-metric space and fuzzy 3-metric space and obtained some common fixed point theorems for three mappings in this setting. Sharma [13] proved common fixed point theorems for commuting maps, thus modified and extended the results due to Fisher [4].

In 2007, Singh et al. [16] introduced the concept of semi-compatibility and weak compatibility in fuzzy -2 , fuzzy-3 metric space.

For the sake of completeness, we recall some definitions and known results in fuzzy, fuzzy -2 , fuzzy- 3 metric spaces.

\section{Preliminaries and definitions}

Definition 2.1. [12] A binary operation $*:[0,1] \times[0,1] \rightarrow[0,1]$ is called a continuous tnorm if it satisfies the following conditions: 
Preeti Sengar, Suman Jain, Aklesh Paria and V.H.Badshah

(i) $*$ is associative and commutative,

(ii) $*$ is continuous,

(iii) $\quad \mathrm{a} * 1=\mathrm{a}$; for all $\mathrm{a} \in[0,1]$

(iv) $\quad \mathrm{a} * \mathrm{~b} \leq \mathrm{c} * \mathrm{~d}$ whenever $\mathrm{a} \leq \mathrm{c}$ and $\mathrm{b} \leq \mathrm{d}$, for all $\mathrm{a}, \mathrm{b}, \mathrm{c}, \mathrm{d} \in[0,1]$.

Example of $\mathrm{t}$-norms are $\mathrm{a} * \mathrm{~b}=\mathrm{ab}$ and $\mathrm{a} * \mathrm{~b}=\min \{\mathrm{a}, \mathrm{b}\}$.

Definition 2.2. [10] The 3-tuple $(X, M, *)$ is called a fuzzy metric space if $X$ is an arbitrary set, $*$ is a continuous t-norm and $\mathrm{M}$ is a fuzzy set in $\mathrm{X}^{2} \times[0, \infty)$ satisfying the following conditions for all $\mathrm{x}, \mathrm{y}, \mathrm{z} \in \mathrm{X}$ and $\mathrm{s}, \mathrm{t}>0$ :

$(\mathrm{FM}-1) \mathrm{M}(\mathrm{x}, \mathrm{y}, 0)=0$;

$(\mathrm{FM}-2) \mathrm{M}(\mathrm{x}, \mathrm{y}, \mathrm{t})=1$, for all $\mathrm{t}>0$ if and only if $\mathrm{x}=\mathrm{y}$;

$(\mathrm{FM}-3) \mathrm{M}(\mathrm{x}, \mathrm{y}, \mathrm{t})=\mathrm{M}(\mathrm{y}, \mathrm{x}, \mathrm{t})$;

$(\mathrm{FM}-4) \mathrm{M}(\mathrm{x}, \mathrm{y}, \mathrm{t}) * \mathrm{M}(\mathrm{y}, \mathrm{z}, \mathrm{s}) \geq \mathrm{M}(\mathrm{x}, \mathrm{z}, \mathrm{t}+\mathrm{s})$

$(\mathrm{FM}-5) \mathrm{M}(\mathrm{x}, \mathrm{y}, \cdot):[0, \infty) \rightarrow[0,1]$ is left continuous.

Note that $\mathrm{M}(\mathrm{x}, \mathrm{y}, \mathrm{t})$ can be thought of as the degree of nearness between $\mathrm{x}$ and $\mathrm{y}$ with respect to $t$. We identify $\mathrm{x}=\mathrm{y}$ with $\mathrm{M}(\mathrm{x}, \mathrm{y}, \mathrm{t})=1$, for all $\mathrm{t}>0$.

The following example shows that every metric space induces a fuzzy metric space.

Example 2.1. [10] Let $(X, d)$ be a metric space. Define $a * b=\min \{a, b\}$ and for all $x, y \in$ $\mathrm{X}, \mathrm{M}(\mathrm{x}, \mathrm{y}, \mathrm{t})=\frac{\mathrm{t}}{\mathrm{t}+\mathrm{d}(\mathrm{x}, \mathrm{y})}$ for all $\mathrm{t}>0$ and $\mathrm{M}(\mathrm{x}, \mathrm{y}, 0)=0$.Then $(X, M, *)$ is a fuzzy metric space. It is called the fuzzy metric space induced by the metric $\mathrm{d}$.

Definition 2.3. [8] Let $(X, M, *)$ be a fuzzy metric space. A sequence $\left\{x_{n}\right\}$ in $X$ is said to convergent to a point $x \in X$ if $\lim _{n \rightarrow \infty} M\left(x_{n}, x, t\right)=1$ for all $t>0$. Further, the sequence $\left\{x_{n}\right\}$ is said to be Cauchy sequence in $X$, if $\lim _{n \rightarrow \infty} M\left(x_{n}, x_{n+p}, t\right)=1$ for all $t>0$ and $p>$ 0 .The space $\mathrm{X}$ is said to be complete if every Cauchy sequence in it converges to a point of it.

Definition 2.4. [16] A function $M$ is continuous in fuzzy metric space iff whenever $\left\{x_{n}\right\} \rightarrow x$ and $\left\{y_{n}\right\} \rightarrow y$ then $\lim _{n \rightarrow \infty} M\left(x_{n}, y_{n}, t\right)=M(x, y, t)$ for each $t>0$.

Definition 2.5. [3] A binary operation $*:[0,1] \times[0,1] \times[0,1] \rightarrow[0,1]$ is called a continuous t-norm if $([0,1], *)$ is an abelian topological monoid with unit 1 such that $\mathrm{a}_{1} * \mathrm{~b}_{1} * \mathrm{c}_{1} \leq \mathrm{a}_{2} * \mathrm{~b}_{2} * \mathrm{c}_{2} \quad$ whenever $\mathrm{a}_{1} \leq \mathrm{a}_{2}, \mathrm{~b}_{1} \leq \mathrm{b}_{2}, \mathrm{c}_{1} \leq \mathrm{c}_{2}$ for all $\mathrm{a}_{1}, \mathrm{a}_{2}, \mathrm{~b}_{1}, \mathrm{~b}_{2}, \mathrm{c}_{1}$ and $c_{2}$ are in $[0,1]$.

Definition 2.6. [13] The 3-tuple $(X, M, *)$ is called a fuzzy-2 metric space if $X$ is an arbitrary set, $*$ is a continuous t-norm and $\mathrm{M}$ is a fuzzy set in $\mathrm{X}^{3} \times[0, \infty)$ satisfying the following conditions for all $\mathrm{x}, \mathrm{y}, \mathrm{z}, \mathrm{u} \in \mathrm{X}$ and $\mathrm{t}_{1}, \mathrm{t}_{2}, \mathrm{t}_{3}>0$ :

$\left(\mathrm{FM}^{\prime}-1\right) \quad \mathrm{M}(\mathrm{x}, \mathrm{y}, \mathrm{z}, 0)=0$;

$\left(F^{\prime}-2\right) M(x, y, z, t)=1$, for all $t>0$ and when at least two of the three points are equal,

$\left(F M^{\prime}-3\right) M(x, y, z, t)=M(y, x, z, t)=M(z, x, y, t)$;

(Symmetry about three variables) 
Common Fixed Point Theorems in Fuzzy 2 and Fuzzy 3-Metric Spaces

$\left(\mathrm{FM}^{\prime}-4\right) \mathrm{M}\left(\mathrm{x}, \mathrm{y}, \mathrm{z}, \mathrm{t}_{1}+\mathrm{t}_{2}+\mathrm{t}_{3}\right) \geq \mathrm{M}\left(\mathrm{x}, \mathrm{y}, \mathrm{u}, \mathrm{t}_{1}\right) * \mathrm{M}\left(\mathrm{x}, \mathrm{u}, \mathrm{z}, \mathrm{t}_{2}\right) * \mathrm{M}\left(\mathrm{u}, \mathrm{y}, \mathrm{z}, \mathrm{t}_{3}\right)$;

(This corresponds to tetrahedron inequality in 2-metric space).

$\left(\mathrm{FM}^{\prime}-5\right) \mathrm{M}\left(\mathrm{x}, \mathrm{y}, \mathrm{z},{ }^{\cdot}\right):[0, \infty) \rightarrow[0,1]$ is left continuous.

The function value $\mathrm{M}(\mathrm{x}, \mathrm{y}, \mathrm{z}, \mathrm{t})$ may be interpreted as the probability that the area of triangle is less than $\mathrm{t}$.

Definition 2.7. [16] Let $(X, M, *)$ be a fuzzy-2 metric space. A sequence $\left\{x_{n}\right\}$ in $X$ is said to convergent to a point $x \in X \quad \lim _{n \rightarrow \infty} M\left(x_{n}, x, a, t\right)=1$, for all $a \in X, t>0$. Further, the sequence $\left\{x_{n}\right\}$ is said to be Cauchy sequence in $X$, if $\lim _{n \rightarrow \infty} M\left(x_{n}, x_{n+p}, a, t\right)=1$ for all $a \in X, t>0$ and $p>0$. The space $X$ is said to be complete if every Cauchy sequence in it converges to a point of it.

Definition 2.8. [16] A function $M$ is continuous in fuzzy-2 metric space iff whenever $\left\{\mathrm{x}_{\mathrm{n}}\right\} \rightarrow \mathrm{x}$ and $\left\{\mathrm{y}_{\mathrm{n}}\right\} \rightarrow \mathrm{y}$ then $\lim _{\mathrm{n} \rightarrow \infty} \mathrm{M}\left(\mathrm{x}_{\mathrm{n}}, \mathrm{y}_{\mathrm{n}}, \mathrm{a}, \mathrm{t}\right)=\mathrm{M}(\mathrm{x}, \mathrm{y}, \mathrm{a}, \mathrm{t})$ for all $\mathrm{a} \in \mathrm{X}$ and for each $\mathrm{t}>0$.

Definition 2.9. [16] A binary operation $*:[0,1] \times[0,1] \times[0,1] \times[0,1] \rightarrow[0,1]$ is called a continuous t-norm if $([0,1], *)$ is an abelian topological monoid with unit 1 such that $a_{1} * b_{1} * c_{1} * d_{1} \leq a_{2} * b_{2} * c_{2} * d_{2}$ whenever $a_{1} \leq a_{2}, b_{1} \leq b_{2}, c_{1} \leq c_{2}, d_{1} \leq d_{2}$ for all $\mathrm{a}_{1}, \mathrm{a}_{2}, \mathrm{~b}_{1}, \mathrm{~b}_{2}, \mathrm{c}_{1}, \mathrm{c}_{2}, \mathrm{~d}_{1}$ and $\mathrm{d}_{2}$ are in $[0,1]$.

Definition 2.10. [13] The 3-tuple $(X, M, *)$ is called a fuzzy-3 metric space if $x$ is an arbitrary set, $*$ is a continuous t-norm and $\mathrm{M}$ is a fuzzy set in $\mathrm{X}^{4} \times[0, \infty)$ satisfying the following conditions for all $\mathrm{x}, \mathrm{y}, \mathrm{z}, \mathrm{w}, \mathrm{u} \in \mathrm{X}$ and $\mathrm{t}_{1}, \mathrm{t}_{2}, \mathrm{t}_{3}, \mathrm{t}_{4}>0$ :

$(F M "-1) M(x, y, z, w, 0)=0$;

$(F M "-2) M(x, y, z, w, t)=1$, for all $t>0$, iff at-least two of the four points are equal

$\left(F^{\prime \prime}-3\right) M(x, y, z, w, t)=M(y, x, z, w, t)=M(w, z, x, y, t)=\ldots$;

(Symmetry)

$(F M "-4) M\left(x, y, z, t_{1}+t_{2}+t_{3}+t_{4}\right) \geq M\left(x, y, z, u, t_{1}\right) * M\left(x, y, u, w, t_{2}\right) *$

$\mathrm{M}\left(\mathrm{x}, \mathrm{u}, \mathrm{z}, \mathrm{w}, \mathrm{t}_{3}\right) * \mathrm{M}\left(\mathrm{u}, \mathrm{y}, \mathrm{z}, \mathrm{w}, \mathrm{t}_{4}\right)$;

$\left(\mathrm{FM}^{\prime}-5\right) \mathrm{M}\left(\mathrm{x}, \mathrm{y}, \mathrm{z}, \mathrm{w},{ }^{\circ}\right):[0, \infty) \rightarrow[0,1]$ is left continuous.

Definition 2.11. [16] Let $(X, M, *)$ be a fuzzy-3 metric space. A sequence $\left\{x_{n}\right\}$ in $X$ is said to convergent to a point $x \in X$ if

$$
\lim _{n \rightarrow \infty} M\left(x_{n}, x, a, b, t\right)=1,
$$

for all $a, b \in X, t>0$. Further, the sequence $\left\{x_{n}\right\}$ is said to be Cauchy sequence in $X$, if $\lim _{n \rightarrow \infty} M\left(x_{n}, x_{n+p}, a, b, t\right)=1$ for all $a, b \in X, t>0$ and $p>0$. The space $X$ is said to be complete if every Cauchy sequence in it converges to a point of it.

Definition 2.12. [16] A function $M$ is continuous in fuzzy-3 metric space iff whenever $\left\{\mathrm{x}_{\mathrm{n}}\right\} \rightarrow \mathrm{x}$ and $\left\{\mathrm{y}_{\mathrm{n}}\right\} \rightarrow \mathrm{y}$ then $\lim _{\mathrm{n} \rightarrow \infty} \mathrm{M}\left(\mathrm{x}_{\mathrm{n}}, \mathrm{y}_{\mathrm{n}}, \mathrm{a}, \mathrm{b}, \mathrm{t}\right)=\mathrm{M}(\mathrm{x}, \mathrm{y}, \mathrm{a}, \mathrm{b}, \mathrm{t})$ for all $\mathrm{a}, \mathrm{b} \in \mathrm{X}$ and for each $t>0$.

Definition 2.13. [11] Let A and B mappings from a fuzzy metric space, $(X, M, *)$ into itself. The mappings are said to be compatible if 
Preeti Sengar, Suman Jain, Aklesh Paria and V.H.Badshah

$\lim _{n \rightarrow \infty} M\left(A B x_{n}, B A x_{n}, t\right)=1$ for all $t>0$, whenever $\left\{x_{n}\right\}$ is a sequence in $X$ such that $\lim _{\mathrm{n} \rightarrow \infty} \mathrm{Ax}_{\mathrm{n}}=\lim _{\mathrm{n} \rightarrow \infty} \mathrm{Bx}_{\mathrm{n}}=\mathrm{x}$ for some $\mathrm{x} \in \mathrm{X}$.

Singh et al.[16] extend this concept in fuzzy-2 metric spaces and in fuzzy-3 metric spaces as follows:

Definition 2.14. [16] Let A and B mappings from a fuzzy-2 metric space, $(X, M, *)$ into itself. The mappings are said to be compatible if $\lim _{n \rightarrow \infty} M\left(A B x_{n}, B A x_{n}, a, t\right)=1$ for all $t>0$ and $a \in X$, whenever $\left\{x_{n}\right\}$ is a sequence in $X$ such that $\lim _{n \rightarrow \infty} A x_{n}=\lim _{n \rightarrow \infty} B x_{n}=x$ for some $\mathrm{x} \in \mathrm{X}$.

Definition 2.15. [16] Let A and B mappings from a fuzzy-3 metric space, $(X, M, *)$ into itself. The mappings are said to be compatible if $\lim _{n \rightarrow \infty} M\left(\mathrm{ABx}_{\mathrm{n}}, \mathrm{BAx}_{\mathrm{n}}, \mathrm{a}, \mathrm{b}, \mathrm{t}\right)=1$ for all $\mathrm{t}>0$ and for all $\mathrm{a}, \mathrm{b} \in \mathrm{X}$, whenever $\left\{\mathrm{x}_{\mathrm{n}}\right\}$ is a sequence in $\mathrm{X}$ such that

$\lim _{n \rightarrow \infty} A x_{n}=\lim _{n \rightarrow \infty} B x_{n}=x$ for some $x \in X$.

Definition 2.16. [17] Two self maps A and B of a fuzzy metric space (X, M, *) are said to be weak compatible if they commute at their coincidence points, that is $\mathrm{Ax}=\mathrm{Bx}$ implies $\mathrm{ABx}=\mathrm{BAx}$.

Definition 2.17. [18] Let A and B mappings from a fuzzy metric space (X, M, *) into itself. The mappings are said to be semi-compatible if $\lim _{\mathrm{n} \rightarrow \infty} \mathrm{M}\left(\mathrm{ABx}_{\mathrm{n}}, \mathrm{Bx}, \mathrm{t}\right)=1$, for all $t>0$, whenever $\left\{x_{n}\right\}$ is a sequence in $X$ such that $\lim _{n \rightarrow \infty} A x_{n}=\lim _{n \rightarrow \infty} B x_{n}=x$ for some $\mathrm{x} \in \mathrm{X}$.

Singh et al.[16] extend this concept in fuzzy- 2 metric spaces and in fuzzy-3 metric spaces as follows:

Definition 2.18. [16] Let A and B mappings from a fuzzy-2 metric space $(X, M, *)$ into itself. The mappings are said to be semi-compatible if

$\lim _{n \rightarrow \infty} M\left(A B x_{n}, B x, a, t\right)=1$ for all $t>0$ and $a \in X$, whenever $\left\{x_{n}\right\}$ is a sequence in $X$ such that $\lim _{\mathrm{n} \rightarrow \infty} \mathrm{Ax}_{\mathrm{n}}=\lim _{\mathrm{n} \rightarrow \infty} \mathrm{Bx}_{\mathrm{n}}=\mathrm{x}$ for some $\mathrm{x} \in \mathrm{X}$.

Definition 2.19. [16] Let A and B mappings from a fuzzy-3 metric space (X, M, *) into itself. The mappings are said to be semi-compatible if

$\lim _{\mathrm{n} \rightarrow \infty} \mathrm{M}(\mathrm{ABx}, \mathrm{Bx}, \mathrm{a}, \mathrm{b}, \mathrm{t})=1$ for all $\mathrm{t}>0$ and $\mathrm{a}, \mathrm{b} \in \mathrm{X}$, whenever $\left\{\mathrm{x}_{\mathrm{n}}\right\}$ is a sequence in $X$ such that $\lim _{n \rightarrow \infty} A x_{n}=\lim _{n \rightarrow \infty} B x_{n}=x$ for some $x \in X$.

Definition 2.20. [3] A Pair (A, S) of self maps of a fuzzy metric space (X, M, *) is said to be reciprocal continuous if $\lim _{\mathrm{n} \rightarrow \infty} \mathrm{ASx}_{\mathrm{n}}=\mathrm{Ax}$ and $\lim _{\mathrm{n} \rightarrow \infty} \mathrm{SAx}_{\mathrm{n}}=\mathrm{Sx}$, whenever there exist a sequence $\left\{x_{n}\right\}$ such that $\lim _{n \rightarrow \infty} A x_{n}=\lim _{n \rightarrow \infty} S_{n}=x$ for some $x \in X$.

In a similar manner, we extend the concept of reciprocal continuity in fuzzy-2 metric spaces and fuzzy-3 metric spaces as follows:

Definition 2.21. A Pair (A, S) of self maps of a fuzzy-2 metric space $(X, M, *)$ is said to be reciprocal continuous if $\lim _{\mathrm{n} \rightarrow \infty} \mathrm{ASx}_{\mathrm{n}}=\mathrm{Ax}$ and $\lim _{\mathrm{n} \rightarrow \infty} \mathrm{SAx}_{\mathrm{n}}=\mathrm{Sx}$, whenever there exist a sequence $\left\{x_{n}\right\}$ such that $\lim _{n \rightarrow \infty} A x_{n}=\lim _{n \rightarrow \infty} S x_{n}=x$ for some $x \in X$. 
Common Fixed Point Theorems in Fuzzy 2 and Fuzzy 3-Metric Spaces

Definition 2.22. A Pair (A, S) of self maps of a fuzzy-3 metric space (X, M, *) is said to be reciprocal continuous if $\lim _{n \rightarrow \infty} \mathrm{ASx}_{\mathrm{n}}=\mathrm{Ax}$ and $\lim _{\mathrm{n} \rightarrow \infty} \mathrm{SAx}_{\mathrm{n}}=\mathrm{Sx}$, whenever there exist a sequence $\left\{x_{n}\right\}$ such that $\lim _{n \rightarrow \infty} A x_{n}=\lim _{n \rightarrow \infty} S x_{n}=x$ for some $x \in X$.

Example 2.2. $\operatorname{Let}(X, M, *)$ be a fuzzy metric space, where $X=[0,4]$. Define self maps A and $\mathrm{B}$ on $\mathrm{X}$ as follows

$$
A x=\left\{\begin{array}{ll}
5 x-4, & \text { if } x \in[0,2] \\
x-3, & \text { if } x \in(2,4]
\end{array}\right\}, B x=\left\{\begin{array}{cc}
x^{2}, & \text { if } x \in[0,2] \\
3 x, & \text { otherwise }
\end{array}\right\}
$$

$x \in[0,4]$.Define a sequence $x_{n}=\left(1-\frac{1}{n}\right)$;

Then $\lim _{n \rightarrow \infty} \mathrm{ABx}_{\mathrm{n}}=\lim _{\mathrm{n} \rightarrow \infty} \mathrm{A}\left(1-\frac{1}{\mathrm{n}}\right)^{2}=\lim _{\mathrm{n} \rightarrow \infty} 5\left(1-\frac{1}{\mathrm{n}}\right)-4=1=\mathrm{Ax}$.

$\lim _{n \rightarrow \infty} A_{n}=\lim _{n \rightarrow \infty} 5\left(1-\frac{1}{n}\right)-4=1$

$\lim _{n \rightarrow \infty} \mathrm{BAx}_{\mathrm{n}}=\lim _{\mathrm{n} \rightarrow \infty} \mathrm{B}\left(5\left(1-\frac{1}{\mathrm{n}}\right)-4\right)$

$$
=\lim _{n \rightarrow \infty} B\left(1-\frac{5}{n}\right)=\lim _{n \rightarrow \infty}\left(1-\frac{5}{n}\right)^{2}=1=B x .
$$

$\lim _{n \rightarrow \infty} B_{x_{n}}=\lim _{n \rightarrow \infty}\left(1-\frac{1}{n}\right)^{2}=1$

$\lim _{n \rightarrow \infty} A B x_{n}=A x$ and $\lim _{n \rightarrow \infty} B A x_{n}=B x$.

Thus pair $(\mathrm{A}, \mathrm{B})$ is reciprocally continuous.

\section{Main results}

Theorem 3.1. Let $(X, M, *)$ be a complete fuzzy- 2 metric space and $t * t \geq t$ for all $\mathrm{t} \in[0,1]$ and let $\mathrm{A}, \mathrm{B}, \mathrm{S}$ and $\mathrm{T}$ be self maps of $\mathrm{X}$ such that

(3.1.1) $\mathrm{A}(\mathrm{X}) \cup \mathrm{B}(\mathrm{X}) \subseteq \mathrm{S}(\mathrm{X}) \cap \mathrm{T}(\mathrm{X})$,

(3.1.2) Pairs (A, T) and $(B, S)$ are semi compatible and are reciprocal continuous,

(3.1.3) $\mathrm{aM}(\mathrm{Tx}, \mathrm{Sy}, \mathrm{v}, \mathrm{t})+\mathrm{bM}(\mathrm{Tx}, \mathrm{Ax}, \mathrm{v}, \mathrm{t})+\mathrm{cM}(\mathrm{Sy}, \mathrm{By}, \mathrm{v}, \mathrm{t})$

$$
+\max \{\mathrm{M}(\mathrm{Ax}, \mathrm{Sy}, \mathrm{v}, \mathrm{t}), \mathrm{M}(\mathrm{By}, \mathrm{Tx}, \mathrm{v}, \mathrm{t})\} \leq \mathrm{qM}(\mathrm{Ax}, \mathrm{By}, \mathrm{v}, \mathrm{t})
$$

for all $\mathrm{x}, \mathrm{y} \in \mathrm{X}$, where $\mathrm{a}, \mathrm{b}, \mathrm{c} \geq 0, \mathrm{q}>0$ with $\mathrm{q}<(\mathrm{a}+\mathrm{b}+\mathrm{c}+1)$.

Then A, B, S and T have a unique common fixed point.

Proof: Let $\mathrm{x}_{\mathrm{o}} \in \mathrm{X}$ be any arbitrary point.

Since $A(X) \subseteq S(X)$, there is a point $x_{1} \in X$ such that $A x_{0}=S x_{1}$.

Again since $\mathrm{B}(\mathrm{X}) \subseteq \mathrm{T}(\mathrm{X})$ for this $\mathrm{x}_{1}$ there is a point $\mathrm{x}_{2} \in \mathrm{X}$ such that $\mathrm{Bx}_{1}=\mathrm{Tx}_{2}$.

Inductively, we construct a sequence $\left\{\mathrm{y}_{2 \mathrm{n}}\right\}$ in $\mathrm{X}$ such that

$$
\mathrm{y}_{2 \mathrm{n}}=\mathrm{Ax}_{2 \mathrm{n}}=\mathrm{Sx}_{2 \mathrm{n}+1} \text { and } \mathrm{y}_{2 \mathrm{n}+1}=\mathrm{Bx}_{2 \mathrm{n}+1}=\mathrm{Tx}_{2 \mathrm{n}+2} \text {, for all } \mathrm{n}=0,1,2, \ldots
$$

Let $\mathrm{M}_{2 \mathrm{n}}=\mathrm{M}\left(\mathrm{y}_{2 \mathrm{n}}, \mathrm{y}_{2 \mathrm{n}+1}, \mathrm{t}\right)<1$ for all $\mathrm{n}$.

Putting $\mathrm{x}=\mathrm{x}_{2 \mathrm{n}}, \mathrm{y}=\mathrm{x}_{2 \mathrm{n}+1}$ in equation (3.1.3), we get

$\mathrm{aM}\left(\mathrm{Tx}_{2 \mathrm{n}}, \mathrm{Sx}_{2 \mathrm{n}+1}, \mathrm{v}, \mathrm{t}\right)+\mathrm{bM}\left(\mathrm{Tx}_{2 \mathrm{n}}, \mathrm{Ax}_{2 \mathrm{n}}, \mathrm{v}, \mathrm{t}\right)+\mathrm{cM}\left(\mathrm{Sx}_{2 \mathrm{n}+1}, \mathrm{Bx} \mathrm{x}_{2 \mathrm{n}+1}, \mathrm{v}, \mathrm{t}\right)$

$$
+\max \left\{\mathrm{M}\left(\mathrm{Ax}_{2 \mathrm{n}}, \mathrm{Sx}_{2 \mathrm{n}+1}, \mathrm{v}, \mathrm{t}\right), \mathrm{M}\left(\mathrm{Bx}_{2 \mathrm{n}+1}, \mathrm{Tx}_{2 \mathrm{n}}, \mathrm{v}, \mathrm{t}\right)\right\} \leq \mathrm{qM}\left(\mathrm{Ax}_{2 \mathrm{n}}, \mathrm{Bx}_{2 \mathrm{n}+1}, \mathrm{v}, \mathrm{t}\right)
$$

$\operatorname{aM}\left(\mathrm{y}_{2 n-1}, \mathrm{y}_{2 n}, \mathrm{v}, \mathrm{t}\right)+\mathrm{bM}\left(\mathrm{y}_{2 \mathrm{n}-1}, \mathrm{y}_{2 \mathrm{n}}, \mathrm{v}, \mathrm{t}\right)+\mathrm{cM}\left(\mathrm{y}_{2 \mathrm{n}}, \mathrm{y}_{2 \mathrm{n}+1}, \mathrm{v}, \mathrm{t}\right)$

$$
+\max \left\{\mathrm{M}\left(\mathrm{y}_{2 \mathrm{n}}, \mathrm{y}_{2 \mathrm{n}}, \mathrm{v}, \mathrm{t}\right), \mathrm{M}\left(\mathrm{y}_{2 \mathrm{n}+1}, \mathrm{y}_{2 \mathrm{n}-1}, \mathrm{v}, \mathrm{t}\right)\right\} \leq \mathrm{qM}\left(\mathrm{y}_{2 \mathrm{n}}, \mathrm{y}_{2 \mathrm{n}+1}, \mathrm{v}, \mathrm{t}\right)
$$

Implies $(\mathrm{a}+\mathrm{b}) \mathrm{M}_{2 \mathrm{n}-1}+\mathrm{cM}_{2 \mathrm{n}}+1 \leq \mathrm{qM}_{2 \mathrm{n}}$

(q- c) $\mathrm{M}_{2 \mathrm{n}} \geq(\mathrm{a}+\mathrm{b}) \mathrm{M}_{2 \mathrm{n}-1}+1$

$(\mathrm{q}-\mathrm{c}) \mathrm{M}_{2 \mathrm{n}}>(\mathrm{a}+\mathrm{b}) \mathrm{M}_{2 \mathrm{n}-1}$

i.e. $\mathrm{M}_{2 \mathrm{n}}>\mathrm{pM}_{2 \mathrm{n}-1}>\mathrm{M}_{2 \mathrm{n}-1,}, \quad$ where $\mathrm{p}=\frac{(\mathrm{a}+\mathrm{b})}{(\mathrm{q}-\mathrm{c})}>1$, 
Preeti Sengar, Suman Jain, Aklesh Paria and V.H.Badshah

Thus $\left\{\mathrm{M}_{2 \mathrm{n}}, \mathrm{n} \geq 0\right\}$ is an increasing sequence of positive real numbers in $[0,1]$ and therefore tends to a limit $\mathrm{L} \leq 1$.

We claim that $L=1$, suppose if not i.e. $L<1$.

If $\mathrm{L}<1$, taking limit as $\mathrm{n} \rightarrow \infty$ in equation (3.1.1.1), we get $\mathrm{L}<\mathrm{L}$, which is a contradiction. Therefore $\mathrm{L}=1$.

Now consider, for any positive integer $\mathrm{p}$,

$\mathrm{M}\left(\mathrm{y}_{2 \mathrm{n}}, \mathrm{y}_{2 \mathrm{n}+\mathrm{p}}, \mathrm{v}, \mathrm{t}\right) \geq \mathrm{M}\left(\mathrm{y}_{2 \mathrm{n}}, \mathrm{y}_{2 \mathrm{n}+1}, \mathrm{v}, \frac{\mathrm{t}}{\mathrm{p}}\right) * \ldots * \mathrm{M}\left(\mathrm{y}_{2 \mathrm{n}+\mathrm{p}-1}, \mathrm{y}_{2 \mathrm{n}+\mathrm{p}}, \mathrm{v}, \frac{\mathrm{t}}{\mathrm{p}}\right)$

Implying $\lim _{\mathrm{n} \rightarrow \infty} \mathrm{M}\left(\mathrm{y}_{2 \mathrm{n}}, \mathrm{y}_{2 \mathrm{n}+\mathrm{p}}, \mathrm{v}, \mathrm{t}\right) \geq 1 * \ldots * 1=1$.

Thus $\left\{y_{2 n}\right\}$ is a Cauchy sequence in $X$. Since $X$ is complete, there exists a point $u \in X$ such that the sequence $\left\{y_{2 n}\right\}$ converges to $u \in X$ and subsequently, the sequences $\left\{\mathrm{Ax}_{2 \mathrm{n}}\right\},\left\{\mathrm{Bx}_{2 \mathrm{n}+1}\right\},\left\{\mathrm{Sx}_{2 \mathrm{n}+1}\right\}$ and $\left\{\mathrm{Tx}_{2 \mathrm{n}+2}\right\}$ and its subsequences also converges to $\mathrm{u} \in \mathrm{X}$.

Now we will show that $\mathrm{u}$ is a common fixed point of $\mathrm{A}, \mathrm{B}, \mathrm{S}$ and $\mathrm{T}$.

Step 1. Since the pair (A, T) is reciprocal continuous, we get $\lim _{n \rightarrow \infty}$ TAx $_{n} \rightarrow T u$, also $\lim _{n \rightarrow \infty}$ TTx $_{n} \rightarrow T u$,

and since pair $(\mathrm{A}, \mathrm{T})$ is semi compatible, we have $\lim _{\mathrm{n} \rightarrow \infty} \mathrm{ATx}_{\mathrm{n}} \rightarrow \mathrm{Tu}$.

Now we put $\mathrm{x}=\mathrm{Tx}_{\mathrm{n}}, \mathrm{y}=\mathrm{x}_{\mathrm{n}}$ in equation (3.1.3), we get

$\operatorname{aM}\left(\mathrm{TTx}_{\mathrm{n}}, \mathrm{Sx}_{\mathrm{n}}, \mathrm{v}, \mathrm{t}\right)+\mathrm{bM}\left(\mathrm{TTx}_{\mathrm{n}}, \mathrm{ATx}_{\mathrm{n}}, \mathrm{v}, \mathrm{t}\right)+\mathrm{cM}\left(\mathrm{Sx}_{\mathrm{n}}, \mathrm{Bx}_{\mathrm{n}}, \mathrm{v}, \mathrm{t}\right)$ $+\max \left\{\mathrm{M}\left(\mathrm{ATx}_{\mathrm{n}}, \mathrm{Sx}_{\mathrm{n}}, \mathrm{v}, \mathrm{t}\right), \mathrm{M}\left(\mathrm{Bx}_{\mathrm{n}}, \mathrm{TTx}_{\mathrm{n}}, \mathrm{v}, \mathrm{t}\right)\right\} \leq \mathrm{qM}\left(\mathrm{ATx}_{\mathrm{n}}, \mathrm{Bx}_{\mathrm{n}}, \mathrm{v}, \mathrm{t}\right)$

Taking limit as $n \rightarrow \infty$, we get

$\mathrm{aM}(\mathrm{Tu}, \mathrm{u}, \mathrm{v}, \mathrm{t})+\mathrm{bM}(\mathrm{u}, \mathrm{Tu}, \mathrm{v}, \mathrm{t})+\mathrm{cM}(\mathrm{u}, \mathrm{u}, \mathrm{v}, \mathrm{t})$

$+\max \{\mathrm{M}(\mathrm{Tu}, \mathrm{u}, \mathrm{v}, \mathrm{t}), \mathrm{M}(\mathrm{u}, \mathrm{Tu}, \mathrm{v}, \mathrm{t})\} \leq \mathrm{qM}(\mathrm{Tu}, \mathrm{u}, \mathrm{v}, \mathrm{t})$

$\mathrm{aM}(\mathrm{Tu}, \mathrm{u}, \mathrm{v}, \mathrm{t})+\mathrm{bM}(\mathrm{u}, \mathrm{Tu}, \mathrm{v}, \mathrm{t})+\mathrm{c}+\mathrm{M}(\mathrm{Tu}, \mathrm{u}, \mathrm{v}, \mathrm{t}) \leq \mathrm{qM}(\mathrm{Tu}, \mathrm{u}, \mathrm{v}, \mathrm{t})$

this gives $c \leq(q-a-b-1) M(T u, u, v, t)$

i.e. $M(T u, u, v, t) \geq\left(\frac{c}{q-a-b-1}\right)>1$,

Thus $\mathrm{Tu}=\mathrm{u}$.

Step 2. Put $x=u$ and $y=x_{n}$, in equation (3.1.3), we get

$\operatorname{aM}\left(\mathrm{Tu}, \mathrm{Sx}_{\mathrm{n}}, \mathrm{v}, \mathrm{t}\right)+\mathrm{bM}(\mathrm{Tu}, \mathrm{Au}, \mathrm{v}, \mathrm{t})+\mathrm{cM}\left(\mathrm{Sx}_{\mathrm{n}}, \mathrm{Bx}_{\mathrm{n}}, \mathrm{v}, \mathrm{t}\right)$ $+\max \left\{\mathrm{M}\left(\mathrm{Au}, \mathrm{Sx}_{\mathrm{n}}, \mathrm{v}, \mathrm{t}\right), \mathrm{M}\left(\mathrm{Bx}_{\mathrm{n}}, \mathrm{Tu}, \mathrm{v}, \mathrm{t}\right)\right\} \leq \mathrm{qM}\left(\mathrm{Au}, \mathrm{Bx}_{\mathrm{n}}, \mathrm{v}, \mathrm{t}\right)$

Taking limit as $n \rightarrow \infty$, we get

$\mathrm{aM}(\mathrm{u}, \mathrm{u}, \mathrm{v}, \mathrm{t})+\mathrm{bM}(\mathrm{u}, \mathrm{Au}, \mathrm{v}, \mathrm{t})+\mathrm{cM}(\mathrm{u}, \mathrm{u}, \mathrm{v}, \mathrm{t})$

$+\max \{\mathrm{M}(\mathrm{Au}, \mathrm{u}, \mathrm{v}, \mathrm{t}), \mathrm{M}(\mathrm{u}, \mathrm{u}, \mathrm{v}, \mathrm{t})\} \leq \mathrm{qM}(\mathrm{Au}, \mathrm{u}, \mathrm{v}, \mathrm{t})$

$\mathrm{a}+\mathrm{bM}(\mathrm{u}, \mathrm{Au}, \mathrm{v}, \mathrm{t})+\mathrm{c}+\max \{\mathrm{M}(\mathrm{Au}, \mathrm{u}, \mathrm{v}, \mathrm{t}), 1\} \leq \mathrm{qM}(\mathrm{Au}, \mathrm{u}, \mathrm{v}, \mathrm{t})$

Implies $(\mathrm{a}+\mathrm{c}+1) \leq(\mathrm{q}-\mathrm{b}) \mathrm{M}(\mathrm{Au}, \mathrm{u}, \mathrm{v}, \mathrm{t})$

i.e. $\quad \mathrm{M}(\mathrm{Au}, \mathrm{u}, \mathrm{v}, \mathrm{t}) \geq\left(\frac{\mathrm{a}+\mathrm{c}+1}{\mathrm{q}-\mathrm{b}}\right)>1$,

This gives $\mathrm{Au}=\mathrm{u}$. Hence $\mathrm{Au}=\mathrm{u}=\mathrm{Tu}$.

Step 3. Since the pair $(B, S)$ is reciprocal continuous.

In this case, $\lim _{n \rightarrow \infty} \mathrm{SBx}_{\mathrm{n}} \rightarrow \mathrm{Su}$, also $\lim _{\mathrm{n} \rightarrow \infty} \mathrm{SSx}_{\mathrm{n}} \rightarrow \mathrm{Su}$,

Since $(B, S)$ is semi compatible, we have $\lim _{n \rightarrow \infty} B_{S x_{n}} \rightarrow S u$.

Let $\mathrm{x}=\mathrm{x}_{\mathrm{n}}, \mathrm{y}=\mathrm{Sx}_{\mathrm{n}}$ in equation (3.1.3), we get

$\mathrm{aM}\left(\mathrm{Tx}_{\mathrm{n}}, \mathrm{SSx}_{\mathrm{n}}, \mathrm{v}, \mathrm{t}\right)+\mathrm{bM}\left(\mathrm{Tx}_{\mathrm{n}}, \mathrm{Ax}_{\mathrm{n}}, \mathrm{v}, \mathrm{t}\right)+\mathrm{cM}\left(\mathrm{SSx}_{\mathrm{n}}, \mathrm{BSx}_{\mathrm{n}}, \mathrm{v}, \mathrm{t}\right)$ 
Common Fixed Point Theorems in Fuzzy 2 and Fuzzy 3-Metric Spaces

Taking limit as $n \rightarrow \infty$, we get

$$
+\max \left\{\mathrm{M}\left(\mathrm{Ax}_{\mathrm{n}}, \mathrm{SSx}_{\mathrm{n}}, \mathrm{v}, \mathrm{t}\right), \mathrm{M}\left(\mathrm{BSx}_{\mathrm{n}}, \mathrm{Tx}_{\mathrm{n}}, \mathrm{v}, \mathrm{t}\right)\right\} \leq \mathrm{qM}\left(\mathrm{Ax}_{\mathrm{n}}, \mathrm{BSx}_{\mathrm{n}}, \mathrm{v}, \mathrm{t}\right)
$$

$\mathrm{aM}(\mathrm{u}, \mathrm{Su}, \mathrm{v}, \mathrm{t})+\mathrm{bM}(\mathrm{u}, \mathrm{u}, \mathrm{v}, \mathrm{t})+\mathrm{cM}(\mathrm{Su}, \mathrm{Su}, \mathrm{v}, \mathrm{t})$

$$
+\max \{\mathrm{M}(\mathrm{u}, \mathrm{Su}, \mathrm{v}, \mathrm{t}), \mathrm{M}(\mathrm{Su}, \mathrm{u}, \mathrm{v}, \mathrm{t})\} \leq \mathrm{qM}(\mathrm{u}, \mathrm{Su}, \mathrm{v}, \mathrm{t})
$$

Implies $\quad \mathrm{aM}(\mathrm{u}, \mathrm{Su}, \mathrm{v}, \mathrm{t})+\mathrm{b}+\mathrm{c}+\mathrm{M}(\mathrm{u}, \mathrm{Su}, \mathrm{v}, \mathrm{t}) \leq \mathrm{qM}(\mathrm{u}, \mathrm{Su}, \mathrm{v}, \mathrm{t})$

$$
(\mathrm{b}+\mathrm{c}) \leq(\mathrm{q}-\mathrm{a}-1) \mathrm{M}(\mathrm{u}, \mathrm{Su}, \mathrm{v}, \mathrm{t})
$$

i.e. $\quad M(u, S u, v, t) \geq\left(\frac{b+c}{q-a-1}\right)>1$

This gives $\mathrm{Su}=\mathrm{u}$. Hence $\mathrm{Au}=\mathrm{Su}=\mathrm{u}=\mathrm{Tu}$.

$\underline{\text { Step 4. }}$ Put $x=x_{n}, y=u$ in equation (3.1.3), we get $\mathrm{aM}\left(\mathrm{Tx}_{\mathrm{n}}, \mathrm{Su}, \mathrm{v}, \mathrm{t}\right)+\mathrm{bM}\left(\mathrm{Tx}_{\mathrm{n}}, \mathrm{Ax}_{\mathrm{n}}, \mathrm{v}, \mathrm{t}\right)+\mathrm{cM}(\mathrm{Su}, \mathrm{Bu}, \mathrm{v}, \mathrm{t})$ $+\max \left\{\mathrm{M}\left(\mathrm{Ax}_{\mathrm{n}}, \mathrm{Su}, \mathrm{v}, \mathrm{t}\right), \mathrm{M}\left(\mathrm{Bu}, \mathrm{Tx}_{\mathrm{n}}, \mathrm{v}, \mathrm{t}\right)\right\} \leq \mathrm{qM}\left(\mathrm{Ax}_{\mathrm{n}}, \mathrm{Bu}, \mathrm{v}, \mathrm{t}\right)$

Taking limit as $n \rightarrow \infty$, we get

$\mathrm{aM}(\mathrm{u}, \mathrm{u}, \mathrm{v}, \mathrm{t})+\mathrm{bM}(\mathrm{u}, \mathrm{u}, \mathrm{v}, \mathrm{t})+\mathrm{cM}(\mathrm{u}, \mathrm{Bu}, \mathrm{v}, \mathrm{t})$ $+\max \{\mathrm{M}(\mathrm{u}, \mathrm{u}, \mathrm{v}, \mathrm{t}), \mathrm{M}(\mathrm{Bu}, \mathrm{u}, \mathrm{v}, \mathrm{t})\} \leq \mathrm{qM}(\mathrm{u}, \mathrm{Bu}, \mathrm{v}, \mathrm{t})$

$\mathrm{a}+\mathrm{b}+\mathrm{cM}(\mathrm{u}, \mathrm{Bu}, \mathrm{v}, \mathrm{t})+1 \leq \mathrm{qM}(\mathrm{u}, \mathrm{Bu}, \mathrm{v}, \mathrm{t})$

Implies $(a+b+1) \leq(q-c) M(u, B u, v, t)$

i.e. $M(u, B u, v, t) \geq\left(\frac{a+b+1}{q-c}\right)>1$, this gives $B u=u$.

Hence $\mathrm{u}=\mathrm{Au}=\mathrm{Su}=\mathrm{Bu}=\mathrm{Tu}$ is a common fixed point of $\mathrm{A}, \mathrm{B}, \mathrm{S}$ and $\mathrm{T}$.

Uniqueness. Let $\mathrm{z} \neq \mathrm{u}$ be another common fixed point of $\mathrm{A}, \mathrm{B}, \mathrm{S}$ and $\mathrm{T}$, then

$\mathrm{Az}=\mathrm{Bz}=\mathrm{Sz}=\mathrm{Tz}=\mathrm{z}$.

Put $x=u$ and $y=z$ in equation (3.1.3), we get

$\mathrm{aM}(\mathrm{Tu}, \mathrm{Sz}, \mathrm{v}, \mathrm{t})+\mathrm{bM}(\mathrm{Tu}, \mathrm{Au}, \mathrm{v}, \mathrm{t})+\mathrm{cM}(\mathrm{Sz}, \mathrm{Bz}, \mathrm{v}, \mathrm{t})$

$$
+\max \{\mathrm{M}(\mathrm{Au}, \mathrm{Sz}, \mathrm{v}, \mathrm{t}), \mathrm{M}(\mathrm{Bz}, \mathrm{Tu}, \mathrm{v}, \mathrm{t})\} \leq \mathrm{qM}(\mathrm{Au}, \mathrm{Bz}, \mathrm{v}, \mathrm{t})
$$

$\mathrm{aM}(\mathrm{u}, \mathrm{z}, \mathrm{v}, \mathrm{t})+\mathrm{bM}(\mathrm{u}, \mathrm{u}, \mathrm{v}, \mathrm{t})+\mathrm{cM}(\mathrm{z}, \mathrm{z}, \mathrm{v}, \mathrm{t})$

$$
+\max \{\mathrm{M}(\mathrm{u}, \mathrm{z}, \mathrm{v}, \mathrm{t}), \mathrm{M}(\mathrm{z}, \mathrm{u}, \mathrm{v}, \mathrm{t})\} \leq \mathrm{qM}(\mathrm{u}, \mathrm{z}, \mathrm{v}, \mathrm{t})
$$

$\mathrm{aM}(\mathrm{u}, \mathrm{z}, \mathrm{v}, \mathrm{t})+\mathrm{b}+\mathrm{c}+\mathrm{M}(\mathrm{u}, \mathrm{z}, \mathrm{v}, \mathrm{t}) \leq \mathrm{qM}(\mathrm{u}, \mathrm{z}, \mathrm{v}, \mathrm{t})$

Implies $(\mathrm{b}+\mathrm{c}) \leq(\mathrm{q}-\mathrm{a}-1) \mathrm{M}(\mathrm{u}, \mathrm{z}, \mathrm{v}, \mathrm{t})$

i.e. $\quad M(u, z, v, t) \geq\left(\frac{b+c}{q-a-1}\right)>1$

This gives $\mathrm{u}=\mathrm{z}$. Hence $\mathrm{u}$ is a unique common fixed point of A, B, S and T. This completes the proof of the theorem.

Theorem 3.2. Let $(X, M, *)$ be a complete fuzzy- 3 metric space and $t * t \geq t$ for all $t \in[0,1]$ and let $A, B, S$ and $T$ be self maps of $X$ such that

(3.2.1) $\quad \mathrm{A}(\mathrm{X}) \cup \mathrm{B}(\mathrm{X}) \subseteq \mathrm{S}(\mathrm{X}) \cap \mathrm{T}(\mathrm{X})$;

(3.2.2) Pairs $(\mathrm{A}, \mathrm{T})$ and $(\mathrm{B}, \mathrm{S})$ are semi compatible and reciprocal continuous;

(3.2.3) $\quad \mathrm{aM}(\mathrm{Tx}, \mathrm{Sy}, \mathrm{v}, \mathrm{w}, \mathrm{t})+\mathrm{bM}(\mathrm{Tx}, \mathrm{Ax}, \mathrm{v}, \mathrm{w}, \mathrm{t})+\mathrm{cM}(\mathrm{Sy}, \mathrm{By}, \mathrm{v}, \mathrm{w}, \mathrm{t})$ $+\max \{\mathrm{M}(\mathrm{Ax}, \mathrm{Sy}, \mathrm{v}, \mathrm{w}, \mathrm{t}), \mathrm{M}(\mathrm{By}, \mathrm{Tx}, \mathrm{v}, \mathrm{w}, \mathrm{t})\} \leq \mathrm{qM}(\mathrm{Ax}, \mathrm{By}, \mathrm{v}, \mathrm{w}, \mathrm{t})$

for all $\mathrm{x}, \mathrm{y} \in \mathrm{X}$, where $\mathrm{a}, \mathrm{b}, \mathrm{c} \geq 0, \mathrm{q}>0$ with $\mathrm{q}<\mathrm{a}+\mathrm{b}+\mathrm{c}+1$.

Then A, B,S and T have a unique common fixed point.

Proof: Let $\mathrm{x}_{0} \in \mathrm{X}$ be any arbitrary point.

Since $A(X) \subseteq S(X)$, there is a point $x_{1} \in X$ such that $A x_{0}=S x_{1}$.

Again since $\mathrm{B}(\mathrm{X}) \subseteq \mathrm{T}(\mathrm{X})$ for this $\mathrm{x}_{1}$ there is a point $\mathrm{x}_{2} \in \mathrm{X}$ such that $\mathrm{Bx}_{1}=\mathrm{Tx}_{2}$.

Inductively, we construct a sequence $\left\{\mathrm{y}_{2 \mathrm{n}}\right\}$ in $\mathrm{X}$ such that

$$
\mathrm{y}_{2 \mathrm{n}}=\mathrm{Ax}_{2 \mathrm{n}}=\mathrm{Sx}_{2 \mathrm{n}+1} \text { and } \mathrm{y}_{2 \mathrm{n}+1}=\mathrm{Bx}_{2 \mathrm{n}+1}=\mathrm{Tx}_{2 \mathrm{n}+2} \text {, for all } \mathrm{n}=0,1,2, \ldots
$$


Preeti Sengar, Suman Jain, Aklesh Paria and V.H.Badshah

Let $\mathrm{M}_{2 \mathrm{n}}=\mathrm{M}\left(\mathrm{y}_{2 \mathrm{n}}, \mathrm{y}_{2 \mathrm{n}+1}, \mathrm{v}, \mathrm{w}, \mathrm{t}\right)<1$ for all $\mathrm{n}$.

Putting $\mathrm{x}=\mathrm{x}_{2 \mathrm{n}}, \mathrm{y}=\mathrm{x}_{2 \mathrm{n}+1}$ in equation (3.2.3), we get

$\mathrm{aM}\left(\mathrm{Tx}_{2 \mathrm{n}}, \mathrm{Sx}_{2 \mathrm{n}+1}, \mathrm{v}, \mathrm{w}, \mathrm{t}\right)+\mathrm{bM}\left(\mathrm{Tx}_{2 \mathrm{n}}, \mathrm{Ax} \mathrm{x}_{2 \mathrm{n}}, \mathrm{v}, \mathrm{w}, \mathrm{t}\right)+\mathrm{cM}\left(\mathrm{Sx}_{2 \mathrm{n}+1}, \mathrm{Bx}_{2 \mathrm{n}+1}, \mathrm{v}, \mathrm{w}, \mathrm{t}\right)$

$+\max \left\{\mathrm{M}\left(\mathrm{Ax}_{2 \mathrm{n}}, \mathrm{Sx}_{2 \mathrm{n}+1}, \mathrm{v}, \mathrm{w}, \mathrm{t}\right), \mathrm{M}\left(\mathrm{Bx}_{2 \mathrm{n}+1}, \mathrm{Tx}_{2 \mathrm{n}}, \mathrm{v}, \mathrm{w}, \mathrm{t}\right)\right\}$ $\leq \mathrm{qM}\left(\mathrm{Ax}_{2 \mathrm{n}}, \mathrm{Bx}_{2 \mathrm{n}+1}, \mathrm{v}, \mathrm{w}, \mathrm{t}\right)$

$\mathrm{aM}\left(\mathrm{y}_{2 \mathrm{n}-1}, \mathrm{y}_{2 \mathrm{n}}, \mathrm{v}, \mathrm{w}, \mathrm{t}\right)+\mathrm{bM}\left(\mathrm{y}_{2 \mathrm{n}-1}, \mathrm{y}_{2 \mathrm{n}}, \mathrm{v}, \mathrm{w}, \mathrm{t}\right)+\mathrm{cM}\left(\mathrm{y}_{2 \mathrm{n}}, \mathrm{y}_{2 \mathrm{n}+1}, \mathrm{v}, \mathrm{w}, \mathrm{t}\right)+$ $\max \left\{\mathrm{M}\left(\mathrm{y}_{2 \mathrm{n}}, \mathrm{y}_{2 \mathrm{n}}, \mathrm{v}, \mathrm{w}, \mathrm{t}\right), \mathrm{M}\left(\mathrm{y}_{2 \mathrm{n}+1}, \mathrm{y}_{2 \mathrm{n}-1}, \mathrm{v}, \mathrm{w}, \mathrm{t}\right)\right\}$ $\leq \mathrm{qM}\left(\mathrm{y}_{2 \mathrm{n}}, \mathrm{y}_{2 \mathrm{n}+1}, \mathrm{~V}, \mathrm{w}, \mathrm{t}\right)$

Implies $(\mathrm{a}+\mathrm{b}) \mathrm{M}_{2 \mathrm{n}-1}+\mathrm{cM}_{2 \mathrm{n}}+1 \leq \mathrm{qM}_{2 \mathrm{n}}$

(q-c) $M_{2 n} \geq(a+b) M_{2 n-1}+1$

(q-c) $M_{2 n}>(a+b) M_{2 n-1}$

i.e. $M_{2 n}>\mathrm{pM}_{2 \mathrm{n}-1}>\mathrm{M}_{2 \mathrm{n}-1}$, where $\mathrm{p}=\frac{(\mathrm{a}+\mathrm{b})}{(\mathrm{q}-\mathrm{c})}>1$.

Thus $\left\{\mathrm{M}_{2 \mathrm{n}}, \mathrm{n} \geq 0\right\}$ is an increasing sequence of positive real numbers in $[0,1]$ and therefore tends to a limit $\mathrm{L} \leq 1$.

We claim that $\mathrm{L}=1$, suppose if not i.e. $\mathrm{L}<1$.

If $\mathrm{L}<1$, taking limit as $\mathrm{n} \rightarrow \infty$ by equation (3.2.1.1), we get $\mathrm{L}<\mathrm{L}$, which is a contradiction. Therefore $\mathrm{L}=1$.

Now consider, for any positive integer $\mathrm{p}$,

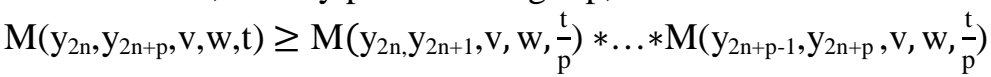

Implying $\lim _{\mathrm{n} \rightarrow \infty} \mathrm{M}\left(\mathrm{y}_{2 \mathrm{n}}, \mathrm{y}_{2 \mathrm{n}+\mathrm{p}}, \mathrm{v}, \mathrm{w}, \mathrm{t}\right) \geq 1 * \ldots * 1=1$

Thus $\left\{y_{2 n}\right\}$ is a Cauchy sequence in $X$. Since $X$ is complete, there is a point $u \in X$ such that $\mathrm{y}_{2 \mathrm{n}}$ converges to $\mathrm{u} \in \mathrm{X}$ and subsequently, the sequences $\left\{\mathrm{Ax}_{2 \mathrm{n}}\right\},\left\{\mathrm{Bx}_{2 \mathrm{n}+1}\right\},\left\{\mathrm{Sx}_{2 \mathrm{n}+1}\right\}$ and $\left\{\mathrm{Tx}_{2 \mathrm{n}+2}\right\}$ and its subsequences also converges to $\mathrm{u} \in \mathrm{X}$.

Now we show that $\mathrm{u}$ is a common fixed point of A, B, S and T.

Step 1. Since the pair $(A, T)$ is reciprocal continuous, we get $\lim _{n \rightarrow \infty} \operatorname{TAx}_{n} \rightarrow T u$, also $\lim _{n \rightarrow \infty} \operatorname{TTx}_{n} \rightarrow T u$.

And since (A, T) is semi compatible, we have $\lim _{n \rightarrow \infty} \operatorname{ATx}_{n} \rightarrow \operatorname{Tu}$.

Now we put $\mathrm{x}=\mathrm{Tx}_{\mathrm{n}}, \mathrm{y}=\mathrm{x}_{\mathrm{n}}$ in equation (3.2.3), we get

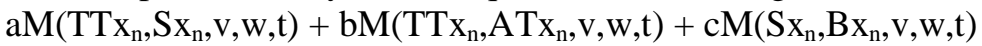

$+\max \left\{\mathrm{M}\left(\mathrm{ATx}_{\mathrm{n}}, \mathrm{Sx}_{\mathrm{n}}, \mathrm{v}, \mathrm{w}, \mathrm{t}\right), \mathrm{M}\left(\mathrm{Bx}_{\mathrm{n}}, \mathrm{TTx}_{\mathrm{n}}, \mathrm{v}, \mathrm{w}, \mathrm{t}\right)\right\} \leq \mathrm{qM}\left(\mathrm{ATx}_{\mathrm{n}}, \mathrm{Bx}_{\mathrm{n}}, \mathrm{v}, \mathrm{w}, \mathrm{t}\right)$

Taking limit as $\mathrm{n} \rightarrow \infty$, we get

$\mathrm{aM}(\mathrm{Tu}, \mathrm{u}, \mathrm{v}, \mathrm{w}, \mathrm{t})+\mathrm{bM}(\mathrm{u}, \mathrm{Tu}, \mathrm{v}, \mathrm{w}, \mathrm{t})+\mathrm{cM}(\mathrm{u}, \mathrm{u}, \mathrm{v}, \mathrm{w}, \mathrm{t})$

$+\max \{\mathrm{M}(\mathrm{Tu}, \mathrm{u}, \mathrm{v}, \mathrm{w}, \mathrm{t}), \mathrm{M}(\mathrm{u}, \mathrm{Tu}, \mathrm{v}, \mathrm{w}, \mathrm{t})\} \leq \mathrm{qM}(\mathrm{Tu}, \mathrm{u}, \mathrm{v}, \mathrm{w}, \mathrm{t})$

$\mathrm{aM}(\mathrm{Tu}, \mathrm{u}, \mathrm{v}, \mathrm{w}, \mathrm{t})+\mathrm{bM}(\mathrm{u}, \mathrm{Tu}, \mathrm{v}, \mathrm{w}, \mathrm{t})+\mathrm{c}+\mathrm{M}(\mathrm{Tu}, \mathrm{u}, \mathrm{v}, \mathrm{w}, \mathrm{t}) \leq \mathrm{qM}(\mathrm{Tu}, \mathrm{u}, \mathrm{v}, \mathrm{w}, \mathrm{t})$

$\begin{aligned} c & \leq(\mathrm{q}-\mathrm{a}-\mathrm{b}-1 \\ \text { i.e } \quad \mathrm{M}(\mathrm{Tu}, \mathrm{u}, \mathrm{v}, \mathrm{w}, \mathrm{t}) & \geq\left(\frac{\mathrm{c}}{\mathrm{q}-\mathrm{a}-\mathrm{b}-1}\right)>1 .\end{aligned}$

This gives $\mathrm{Tu}=\mathrm{u}$.

Step 2. Put $x=u$ and $y=x_{n}$ in equation (3.2.3), we get

$\mathrm{aM}\left(\mathrm{Tu}, \mathrm{Sx}_{\mathrm{n}}, \mathrm{v}, \mathrm{w}, \mathrm{t}\right)+\mathrm{bM}(\mathrm{Tu}, \mathrm{Au}, \mathrm{v}, \mathrm{w}, \mathrm{t})+\mathrm{cM}\left(\mathrm{Sx}_{\mathrm{n}}, \mathrm{Bx}_{\mathrm{n}}, \mathrm{v}, \mathrm{w}, \mathrm{t}\right)$ $+\max \left\{\mathrm{M}\left(\mathrm{Au}, \mathrm{Sx}_{\mathrm{n}}, \mathrm{v}, \mathrm{w}, \mathrm{t}\right), \mathrm{M}\left(\mathrm{Bx}_{\mathrm{n}}, \mathrm{Tu}, \mathrm{v}, \mathrm{w}, \mathrm{t}\right)\right\} \leq \mathrm{qM}\left(\mathrm{Au}, \mathrm{Bx}_{\mathrm{n}}, \mathrm{v}, \mathrm{w}, \mathrm{t}\right)$

Taking limit as $n \rightarrow \infty$, we get

$\mathrm{aM}(\mathrm{u}, \mathrm{u}, \mathrm{v}, \mathrm{w}, \mathrm{t})+\mathrm{bM}(\mathrm{u}, \mathrm{Au}, \mathrm{v}, \mathrm{w}, \mathrm{t})+\mathrm{cM}(\mathrm{u}, \mathrm{u}, \mathrm{v}, \mathrm{w}, \mathrm{t})$ 
Common Fixed Point Theorems in Fuzzy 2 and Fuzzy 3-Metric Spaces

$$
\begin{aligned}
& \quad+\max \{M(A u, u, v, w, t), M(u, u, v, w, t)\} \leq q M(A u, u, v, w, t) \\
& a+b M(u, A u, v, w, t)+c+\max \{M(A u, u, v, w, t), 1\} \leq q M(A u, u, v, w, t) \\
& \text { Implies }(a+c+1) \leq(q-b) M(A u, u, v, w, t) \\
& \text { i.e. } \quad M(A u, u, v, w, t) \geq\left(\frac{a+c+1}{q-b}\right)>1 .
\end{aligned}
$$

This gives $\mathrm{Au}=\mathrm{u}$. Hence $\mathrm{Au}=\mathrm{u}=\mathrm{Tu}$.

Step 3. Since the pair $(B, S)$ is reciprocal continuous, we have

$\varlimsup_{\lim _{n \rightarrow \infty}} S_{B x} \rightarrow$ Su, also $\lim _{n \rightarrow \infty} S x_{n} \rightarrow$ Su.

And since $(\mathrm{B}, \mathrm{S})$ is semi compatible, we have

$$
\lim _{n \rightarrow \infty} B_{S x_{n}} \rightarrow \text { Su. }
$$

Let $\mathrm{x}=\mathrm{x}_{\mathrm{n}}, \mathrm{y}=\mathrm{Sx}_{\mathrm{n}}$ in equation (3.2.3), we get

$$
\begin{aligned}
\mathrm{aM}\left(\mathrm{Tx}_{\mathrm{n}}, \mathrm{SSx}_{\mathrm{n}}, \mathrm{v}, \mathrm{w}, \mathrm{t}\right)+\mathrm{bM}\left(\mathrm{Tx}_{\mathrm{n}}, \mathrm{Ax} \mathrm{x}_{\mathrm{n}}, \mathrm{v}, \mathrm{w}, \mathrm{t}\right)+\mathrm{cM}\left(\mathrm{SSx}_{\mathrm{n}}, \mathrm{BSx}_{\mathrm{n}}, \mathrm{v}, \mathrm{w}, \mathrm{t}\right) \\
+\max \left\{\mathrm{M}\left(\mathrm{Ax}_{\mathrm{n}}, \mathrm{SSx}_{\mathrm{n}}, \mathrm{v}, \mathrm{w}, \mathrm{t}\right), \mathrm{M}\left(\mathrm{BSx}_{\mathrm{n}}, \mathrm{Tx}_{\mathrm{n}}, \mathrm{v}, \mathrm{w}, \mathrm{t}\right)\right\} \\
\leq \mathrm{qM}\left(\mathrm{Ax}_{\mathrm{n}}, \mathrm{BSx}_{\mathrm{n}}, \mathrm{v}, \mathrm{w}, \mathrm{t}\right)
\end{aligned}
$$

Taking limit as $n \rightarrow \infty$, we get

$\mathrm{aM}(\mathrm{u}, \mathrm{Su}, \mathrm{v}, \mathrm{w}, \mathrm{t})+\mathrm{bM}(\mathrm{u}, \mathrm{u}, \mathrm{v}, \mathrm{w}, \mathrm{t})+\mathrm{cM}(\mathrm{Su}, \mathrm{Su}, \mathrm{v}, \mathrm{w}, \mathrm{t})$

$$
+\max \{\mathrm{M}(\mathrm{u}, \mathrm{Su}, \mathrm{v}, \mathrm{w}, \mathrm{t}), \mathrm{M}(\mathrm{Su}, \mathrm{u}, \mathrm{v}, \mathrm{w}, \mathrm{t})\} \leq \mathrm{qM}(\mathrm{u}, \mathrm{Su}, \mathrm{v}, \mathrm{w}, \mathrm{t})
$$

$\mathrm{aM}(\mathrm{u}, \mathrm{Su}, \mathrm{v}, \mathrm{w}, \mathrm{t})+\mathrm{b}+\mathrm{c}+\mathrm{M}(\mathrm{u}, \mathrm{Su}, \mathrm{v}, \mathrm{w}, \mathrm{t}) \leq \mathrm{qM}(\mathrm{u}, \mathrm{Su}, \mathrm{v}, \mathrm{w}, \mathrm{t})$

Implies $\quad(b+c) \leq(q-a-1) M(u, S u, v, w, t)$

Implies $M(u, S u, v, w, t) \geq\left(\frac{b+c}{q-a-1}\right)>1$

This gives $\mathrm{Su}=\mathrm{u}$. Hence $\mathrm{Au}=\mathrm{Su}=\mathrm{u}=\mathrm{Tu}$.

Step 4. Put $x=x_{n}, y=u$ in equation (3.2.3), we get

$\mathrm{aM}\left(\mathrm{Tx}_{\mathrm{n}}, \mathrm{Su}, \mathrm{v}, \mathrm{w}, \mathrm{t}\right)+\mathrm{bM}\left(\mathrm{Tx}_{\mathrm{n}}, \mathrm{Ax} \mathrm{x}_{\mathrm{n}}, \mathrm{v}, \mathrm{w}, \mathrm{t}\right)+\mathrm{cM}(\mathrm{Su}, \mathrm{Bu}, \mathrm{v}, \mathrm{w}, \mathrm{t})$ $+\max \left\{\mathrm{M}\left(\mathrm{Ax}_{\mathrm{n}}, \mathrm{Su}, \mathrm{v}, \mathrm{w}, \mathrm{t}\right), \mathrm{M}\left(\mathrm{Bu}, \mathrm{Tx}_{\mathrm{n}}, \mathrm{v}, \mathrm{w}, \mathrm{t}\right)\right\} \leq \mathrm{qM}\left(\mathrm{Ax} \mathrm{x}_{\mathrm{n}}, \mathrm{Bu}, \mathrm{v}, \mathrm{w}, \mathrm{t}\right)$

Taking limit as $\mathrm{n} \rightarrow \infty$, we get

a $M(u, u, v, w, t)+b M(u, u, v, w, t)+c M(u, B u, v, w, t)$

$$
+\max \{\mathrm{M}(\mathrm{u}, \mathrm{u}, \mathrm{v}, \mathrm{w}, \mathrm{t}), \mathrm{M}(\mathrm{Bu}, \mathrm{u}, \mathrm{v}, \mathrm{w}, \mathrm{t})\} \leq \mathrm{qM}(\mathrm{u}, \mathrm{Bu}, \mathrm{v}, \mathrm{w}, \mathrm{t})
$$

$\mathrm{a}+\mathrm{b}+\mathrm{c} \mathrm{M}(\mathrm{u}, \mathrm{Bu}, \mathrm{v}, \mathrm{w}, \mathrm{t})+1 \leq \mathrm{qM}(\mathrm{u}, \mathrm{Bu}, \mathrm{v}, \mathrm{w}, \mathrm{t})$

Implies $(a+b+1) \leq(q-c) M(u, B u, v, w, t)$

$$
\text { i.e. } \quad M(u, B u, v, w, t) \geq\left(\frac{a+b+1}{q-c}\right)>1 \text {. }
$$

This gives $\mathrm{Bu}=\mathrm{u}$.

Hence $\mathrm{u}=\mathrm{Au}=\mathrm{Su}=\mathrm{Bu}=\mathrm{Tu}$ is a common fixed point of $\mathrm{A}, \mathrm{B}, \mathrm{S}$ and $\mathrm{T}$.

Uniqueness. Let $\mathrm{z} \neq \mathrm{u}$ be another common fixed point of $\mathrm{A}, \mathrm{B}, \mathrm{S}$ and $\mathrm{T}$, then $\mathrm{Az}=\mathrm{Bz}=\mathrm{Sz}=\mathrm{Tz}=\mathrm{z}$.

Put $x=u$ and $y=z$ in equation (3.2.3), we get $\mathrm{aM}(\mathrm{Tu}, \mathrm{Sz}, \mathrm{v}, \mathrm{w}, \mathrm{t})+\mathrm{bM}(\mathrm{Tu}, \mathrm{Au}, \mathrm{v}, \mathrm{w}, \mathrm{t})+\mathrm{cM}(\mathrm{Sz}, \mathrm{Bz}, \mathrm{v}, \mathrm{w}, \mathrm{t})$

$$
+\max \{\mathrm{M}(\mathrm{Au}, \mathrm{Sz}, \mathrm{v}, \mathrm{w}, \mathrm{t}), \mathrm{M}(\mathrm{Bz}, \mathrm{Tu}, \mathrm{v}, \mathrm{w}, \mathrm{t})\} \leq \mathrm{qM}(\mathrm{Au}, \mathrm{Bz}, \mathrm{v}, \mathrm{w}, \mathrm{t})
$$

$\mathrm{aM}(\mathrm{u}, \mathrm{z}, \mathrm{v}, \mathrm{w}, \mathrm{t})+\mathrm{bM}(\mathrm{u}, \mathrm{u}, \mathrm{v}, \mathrm{w}, \mathrm{t})+\mathrm{cM}(\mathrm{z}, \mathrm{z}, \mathrm{v}, \mathrm{w}, \mathrm{t})$

$$
+\max \{\mathrm{M}(\mathrm{u}, \mathrm{z}, \mathrm{v}, \mathrm{w}, \mathrm{t}), \mathrm{M}(\mathrm{z}, \mathrm{u}, \mathrm{v}, \mathrm{w}, \mathrm{t})\} \leq \mathrm{qM}(\mathrm{u}, \mathrm{z}, \mathrm{v}, \mathrm{w}, \mathrm{t})
$$

$\mathrm{aM}(\mathrm{u}, \mathrm{z}, \mathrm{v}, \mathrm{w}, \mathrm{t})+\mathrm{b}+\mathrm{c}+\mathrm{M}(\mathrm{u}, \mathrm{z}, \mathrm{v}, \mathrm{w}, \mathrm{t}) \leq \mathrm{qM}(\mathrm{u}, \mathrm{z}, \mathrm{v}, \mathrm{w}, \mathrm{t})$

Implies $\quad(\mathrm{b}+\mathrm{c}) \leq(\mathrm{q}-\mathrm{a}-1) \mathrm{M}(\mathrm{u}, \mathrm{z}, \mathrm{v}, \mathrm{w}, \mathrm{t})$

$$
\text { i.e. } \quad M(u, z, v, w, t) \geq\left(\frac{b+c}{q-a-1}\right)>1 \text {. }
$$

This gives $\mathrm{u}=\mathrm{z}$. 
Preeti Sengar, Suman Jain, Aklesh Paria and V.H.Badshah

Hence, $\mathrm{u}$ is a unique common fixed point of $\mathrm{A}, \mathrm{B}, \mathrm{S}$ and $\mathrm{T}$. This completes the proof of the theorem.

\section{Conclusion}

Using the notion of semi-compatible and reciprocal continuity of mappings, theorem 3.1 and theorem 3.2 are generalization of some results of Chauhan et al. [1] and Som [19] results of from fuzzy metric spaces to fuzzy 2 , fuzzy 3 - metric spaces.

\section{REFERENCES}

1. M.S.Chauhan, V.H.Badshah and V.Chouhan, Common fixed point of semi compatible maps in fuzzy metric spaces, Kathmandu University Journal of Science, Engineering and Technology, 6(1) (2010) 70-78.

2. Y.J.Cho, Fixed points in fuzzy metric spaces, J. Fuzzy Math., 5(4) (1997) 949-962.

3. K.S.Dersanambika and M.R.Aswathy, Fixed point theorems in fuzzy metric spaces, Int. J. Contemp. Math. Sciences, 6(22) (2011) 1079-1089.

4. B.Fisher, Fixed point on two metric space, Glasnik Mathematical, 16 (36) (1981) 333-337.

5. S.Gahler, 2-metrische Raume und ihre topologische Struktur, Math. Nachr., 26 (1963) 115-148.

6. S.Gahler, Line are 2-normierte, Raume. Math. Nachr., 28 (1964) 1-43.

7. A.George and P.Veeramani, On some results in fuzzy metric spaces, Fuzzy Sets and Systems, 64 (1994) 395-399.

8. M.Grabiec, Fixed points in fuzzy metric spaces, Fuzzy Sets and Systems, 27(3) (1988) 385-389.

9. O.Kaleva, The completion of fuzzy metric spaces, J. Math. Anal. Appl., 109(1) (1985) 194-198.

10. I.Kramosil and J.Michalek, Fuzzy metric and Statistical metric space, Kybernetica, 11 (1975) 336-344.

11. S.N.Mishra, N.Sharma and S.L.Singh, Common fixed points of maps on fuzzy metric spaces, Int. J. Math. Math. Sci., 17(2) (1994) 253-258.

12. B.Schweizer and A.Sklar, Statistical metric spaces, Pacific J. Math., 10 (1960) 313334.

13. P.LSharma, B.K.Sharma and K.Iseki, Contractive type mapping on 2-metric space, Math. Japonica, 21 (1976) 67-70.

14. S.Sharma, On fuzzy metric space, South East Asian Bulletin of Mathematics, 26 (2002) 133-145.

15. B.Singh and M.S.Chauhan, Common fixed points of compatible maps in fuzzy metric spaces, Fuzzy Sets and Systems, 115 (2000) 471-475.

16. B.Singh and S.Jain, Semi-compatibility, compatibility and fixed point theorems in fuzzy metric space, Journal of Chungecheong Math. Soc., 18(1) (2005) 1-22.

17. B.Singh, S.Jain and S.Jain, Fixed point theorems in fuzzy metric spaces, fuzzy-2 metric spaces and fuzzy-3 metric spaces using semi-compatibility, East Asian Math. J., 23(2) (2007) 175-195.

18. T.Som, Some results on common fixed point in fuzzy metric spaces, Soochow J. Mathematics, 33(4) (2007) 553-561.

19. L.A.Zadeh, Fuzzy sets, Inform. and Control, 89 (1965) 338-353. 